\title{
CODIUM TOMENTOSUM VAR. MUCRONATUM ET SON EPIPHYTE AGLAOTHAMNION PSEUDOBYSSOIDES, DEUX NOUVELLES ESPÈCES D'ALGUES BENTHIQUES POUR LA PHYCOFLORE DU MAROC
}

\author{
Mustapha HASSOUN, Ghizlane SALHI, Hajar BOUKSIR, Hanaa MOUSSA, \\ Hassane RIADI \& Mohamed KAZZAZ* \\ Univ. Abdelmalek Essaâdi, Faculté des Sciences de Tétouan, Départ. De Biologie, Groupe Algologie- \\ Mycologie Appliquées (AMA), 93000, BP. 2121, M’hannech II, Tétouan, Maroc. \\ *Corresponding author: seaweedprof@gmail.com
}

Recibido el 5 mayo de 2014, aceptado para su publicación el 6 de junio de 2014

RESUME. Codium tomentosum var. mucronatum et son Epiphyte Aglaothamnion pseudobyssoides, deux nouvelles espèces d'algues benthiques pour la phycoflore du Maroc. Deux algues marines nouvelles pour la phycoflore benthique du Maroc sont décrites. Il s'agit d'une Ulvophyceae: Codium tomentosum var. mucronatum et une Rhodophyceae: Aglaothamnion pseudobyssoides. Les deux espèces sont étudiées en détail et des données écologiques sont ajoutées aux descriptions morphologiques et anatomiques.

Mots clés. Aglaothamnion pseudobyssoides, Codium tomentosum var. mucronatum, Maroc, phycoflore benthique.

ABSTRACT. Codium tomentosum var. mucronatum and Aglaothamnion pseudobyssoides, two new records for Morocco. Two new records (Codium tomentosum var. mucronatum and Aglaothamnion pseudobyssoides) for the benthic seaweeds of Morocco are described. Their geographical distributions, the description and illustration of their macroscopic and microscopic characters and their ecological characteristics are presented and discussed in this work.

Key words. Aglaothamnion pseudobyssoides, Codium tomentosum var. mucronatum, Morocco, new record, seaweed.

\section{INTRODUCTION}

La côte atlantique du Maroc se prolonge sur plus de $3000 \mathrm{~km}$, depuis le rivage de Tanger jusqu'à celui d'EL Gouira. La largeur du plateau continental est variable et peut atteindre $150 \mathrm{Km}$ dans les zones au sud d'Agadir et 50 $\mathrm{Km}$ au nord. La flore marine benthique du Maroc, notamment celle de la côte atlantique recèle une importante richesse en espèces 
d'intérêt économique et écologique. Dès la moitié du $\mathrm{XX}^{\mathrm{ème}}$ siècle, les côtes marocaines ont suscité un intérêt particulier de la part de plusieurs auteurs tels que: Dangeard (1949, 1957), Feldmann (1955), Gayral (1958, 1961) qui s'efforçaient à présenter une synthèse sur la flore algale du littoral Atlantique. D'autres auteurs ont étudié des zones concrètes de la côte marocaine, tels que Esmiole (1962) sur la région de Rabat, Cavassillas (1963) sur la région d'El Harhoura, Salaheddine (1982) sur le tronçon Rabat-Bouznika, Tahi (1983) sur la région Salé-Bouknadel, Berday (1989) sur la région d'El Jadida, Riadi \& Kazzaz (1998), Benhissoune et al. (2001, 2002a-b, 2003).

Toutefois cette richesse est loin d'être complètement inventoriée et de grandes lacunes restent à combler. En effet, plusieurs zones rocheuses, à accès difficile sur l'Atlantique, sont encore non étudiées.

Dans ce travail, les différents critères de détermination des deux espèces sont décrits, illustrés et discutés.

\section{MATÉRIEL ET MÉTHODES}

Le site de collecte est le littoral de Hyayda $\left(35^{\circ} 05^{\prime} 15.7^{\prime \prime} \mathrm{N} 6^{\circ} 12^{\prime} 51.4^{\prime \prime} \mathrm{W}\right)$, au nord du Maroc dans la région de la ville de Larache, qui donne sur l'atlantique. Le site se localise à $12 \mathrm{kms}$ du centre de Larache.

La programmation des dates des sorties d'exploration et collectes est choisie en se rapportant au calendrier des marées. En effet, la collecte des espèces étudiées a été réalisée le 06 septembre 2013. Le matériel utilisé comporte le matériel permettant la collecte et le stockage des échantillons tels que: râteaux, grattoirs, des bocaux en plastique. L'échantillonnage a été accompli à marée basse en parcourant tout le médiolittoral supérieur, moyen et inférieur et l'infralittoral supérieur.

Les échantillons sont directement conservés dans une solution d'eau de mer formolée à $5 \%$.
Une partie bien représentative est sauvegardée dans de l'eau de mer mais au sein d'une enceinte réfrigérée. Les échantillons les plus typiques sont destinés à l'herbier du laboratoire, (HTET $795,796)$, le reste aux études taxonomiques et floristiques.

Pour chaque taxon, la présence et la distribution globale et au Maroc ont été examinées en détail, sur la base des différents inventaires bibliographiques régionaux existants. La réactualisation systématique a été faite en accord avec Algaebase de Guiry \& Guiry (2014). Les données écologiques mentionnées correspondent plutôt aux conditions relatives à nos récoltes et non pas à l'amplitude écologique de l'espèce en Atlantique.

\section{RÉSULTATS}

1. Codium tomentosum var. mucronatum (G.Hamel) Ardré (HTET n 795)

=Codium elongatum var. mucronatum $\mathrm{G}$. Hamel

Distribution. En Espagne (Hamel, 1931; Gorostiaga et al. 2004; Bárbara et al. 2005; Martínez-Gil et al. 2007; Cires Rodríguez \& Cuesta Molinier (2010); Bermejo et al. 2012). France (Bouxin \& Dizerbo, 1971). Portugal (Ardré, 1971; Araújo et al. 2009; Berecibar Zugasti, 2011).

Description. Algue d'un vert foncé, avec un thalle dressé à ramification assez régulièrement dichotome et fastigiée (fig. 1 A). La taille moyenne est en général de 25 à $38 \mathrm{~cm}$, la fronde est généralement aplatie, parfois les proliférations terminales sont un peu cylindriques (fig.1 B). La base discoïdale est formée de nombreux filaments encastrés dans la roche (fig.1 C).

Au microscope le thalle apparaît composé de filaments incolores, ramifiés, enchevêtrés, terminés au niveau de la zone corticale par des utricules larges de 160-300 $\mu \mathrm{m}$ et longues de 


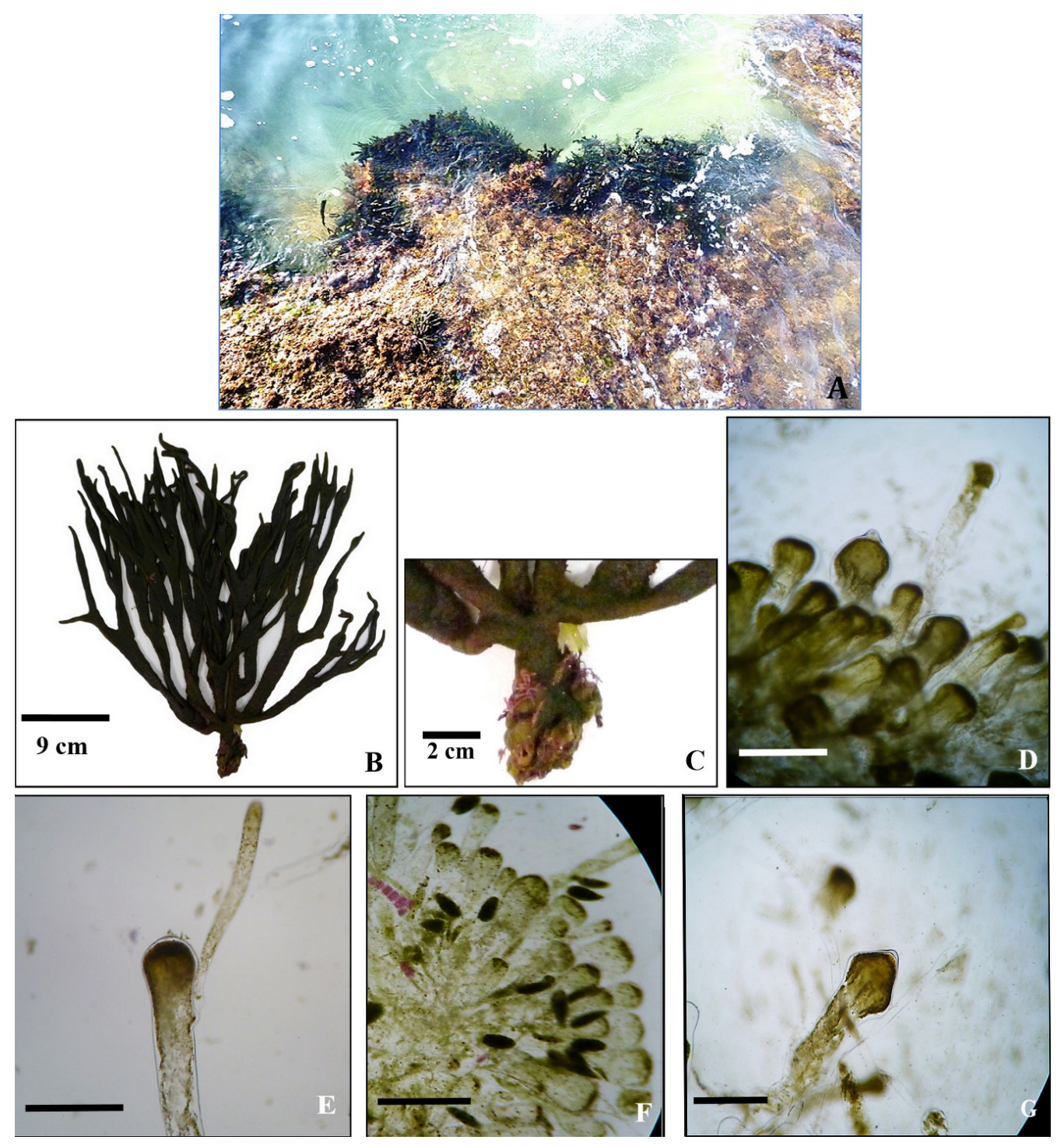

Figure 1. Codium tomentosum var.mucronatum. A. Habitat de l'espèce. B. Aspect général du thalle avec des ramifications assez régulièrement dichotomes. C. Base discoïdale qui sert à la fixation du thalle. D. Utricules. E. Utricule avec un poil hyalin. F. Gamétocystes se forment latéralement sur les utricules. G. Utricule se terminent par un mucron bien visible. La barre d'échelle représente $400 \mu \mathrm{m}$. Codium tomentosum var. mucronatum, A. Habitat of species, in lower intertidal rocky. B. Habit of thallus with dichotomous branching that are compressed at some parts. $\boldsymbol{C}$. Discoid holdfast. D. Utricules of several sizes and forms. $\boldsymbol{E}$. Small utricule with hyaline hair. F. Lateral position of the gametocystes. G. Bigger utricule with pseudomucron. Scale bar represents $400 \mu \mathrm{m}$. 
1000 à $1200 \mu \mathrm{m}$ (fig.1 D), certaines d'entre elles portent un poil hyalin (fig. 1 E). Parfois les utricules sont un peu gonflés en leur sommet et se terminent par un pseudomucron bien visible au microscope (fig.1 G).

On remarque la présence des gamétocystes qui se forment latéralement sur les utricules, larges de 40-55 $\mu \mathrm{m}$ et longs de 100 à $160 \mu \mathrm{m}$ (fig.1 F).

Discussion. Par l'ensemble de ses caractères, cette algue est conforme, en tout point, aux figures présentées par Bárbara (2009) et aux descriptions d'Ardré(1971). Cette algue ne peut être d'aucune façon rattachée au Codium elongatum var. mucronatum, qui n'été d'ailleurs citée qu'en Archipel des Glenan, dont la fronde est comprimée et la largeur des utricules toujours supérieure à $200 \mu \mathrm{m}$ (Hamel, 1931; Bouxin \& Dizerbo, 1971). De même, elle ne peut être confondue avec Codium fragile (Suringar) Hariot, de distribution assez vaste, chez qui la fronde est cylindrique et la largeur des utricules à mucron ne dépasse jamais 100 $\mu \mathrm{m}$ (Gayral, 1966). On outre, C. tomentosum var. murcronatum ne contient pas de véritables utricules mucronés, mais des pseudomucron apicaux gonflés.

Notre algue rappelle aussi Codium decorticatum (Woodward) M. A. Howe dans son aspect général comprimé et aplati, mais s'en différencie par l'absence de mucron et aussi les utricule chez codium decorticatum sont plus grand, pouvant atteindre $500 \mu \mathrm{m}$ de largeur et $1800 \mu \mathrm{m}$ de longueur (Gayral, 1966; Rodríguez et al. 2013).

Écologie et phénologie. Cette algue se développe sur les rochers à basse mer et dans les flaques à mi-marée, parfois fixée au bord des cuvettes de la zone littorale moyenne et inférieure. Elle se trouve en abondance au-dessous des niveaux de la base mer. Les gamétocystes ont été observés en été et au début d'automne. Cette espèce était rencontrée seulement en période estivale et au début de l'automne.

2. Aglaothamnion pseudobyssoides (P. L. Crouan \& H. M. Crouan) Halos (HTET $\mathrm{n}^{\circ} 796$ )

= Callithamnion pseudobyssoides P. L. Crouan \& H. M. Crouan

Callithamnion byssoides var. pseudobyssoides (P.L.Crouan \& H.M.Crouan) Miranda

Distribution. L'espèce a été déjà signalée en Espagne (Valenzuela Miranda, 2002; Bárbara et al. 2005; Peña \& Bárbara, 2008; Secilla, 2012); en France (L'Hardy-Halos \& Rueness, 1990; Valenzuela Miranda, 2005); en Grande-Bretagne (Maggs \& Hommersand, 1993; Hardy \& Guiry, 2003); en Portugal (Araujo et al. 2009).

Description. Algue de petite taille, se présentant sous forme de petites touffes rouges de 0,2-1,5 cm de haut, dépassant rarement $1,5 \mathrm{~cm}$, avec thalle filamenteux et dressé constitué d'un seul axe principale à ramification alterne spiralée (fig.2 A-B). La cortication est totalement absente, même à la base du thalle (fig. 2 C-D).

La fronde est trouvé épiphyte sur Codium tomentosum var. mucronatum, fixée par des rhizoïdes pluricellulaires. Le diamètre des rameaux principaux varie le plus souvent entre 50 et $63,5 \mu \mathrm{m}$, ne dépasse jamais $70 \mu \mathrm{m}$, celui des rameaux secondaires entre 22 et $30 \mu \mathrm{m}$ (fig. 2 E-F) et enfin chez les ramules entre 15 et 20 $\mu \mathrm{m}$. Le diamètre des cellules apicales de l'axe principal est entre 7,5 et $10 \mu \mathrm{m}$ (fig. $2 \mathrm{G}$ ).

L'axe principal présente au niveau des cellules axiales de la base, des rhizoïdes pluricellulaires qui pénètrent à l'intérieur de l'algue support. Ces rhizoïdes ont un diamètre qui varie entre 22 et $25 \mu \mathrm{m}$ (fig. 2-H).

Les tétrasporocystes sont sessiles, ovoïdes ou subsphériques, situés sur le côté internes 

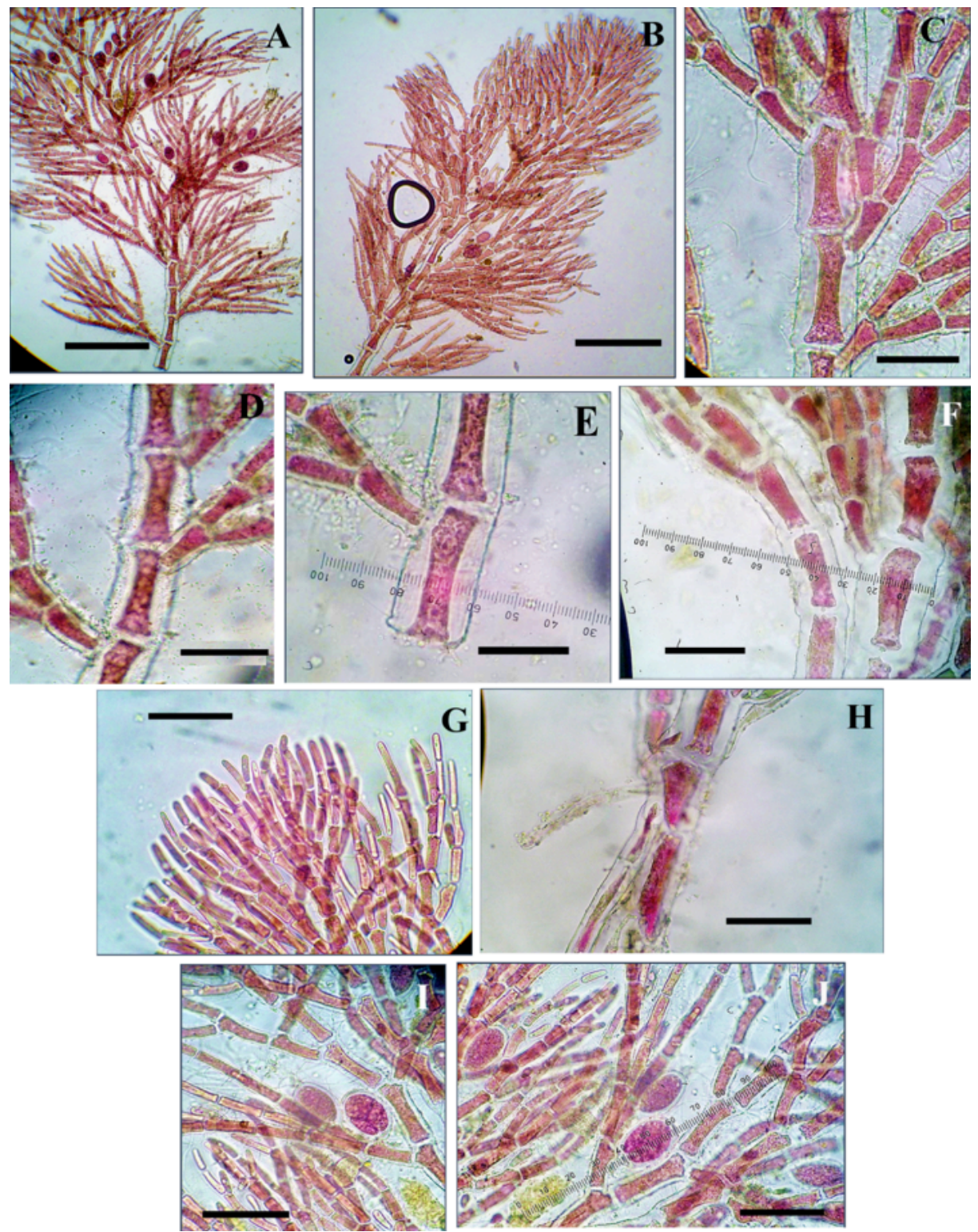

Figure 2: Aglaothamnion pseudobyssoide. A-B. Aspect général du thalle, montrant des ramifications très denses. C-D. Détail de l'axe principal décortiqué, avec ramification spiralée. E-F. Détails d'un rameau principal et rameaux secondaires. G. Cellules apicales du thalle de forme arrondie. H. Détail d'un rhizoïde pluricellulaire. I-J. Rameau présentant des tétrasporocystes sessiles et ovoïdes, situés sur le côté internes des ramules (disposition adaxiale). La barre d'échelle représente $75 \mu \mathrm{m}$. Aglaothamnion pseudobyssoides. $\boldsymbol{A}-\boldsymbol{B}$. Habit of thallus with dense branching. $\boldsymbol{C}-\boldsymbol{D}$. Detail of main axis ecorticated, with a spiral arrangement. $\boldsymbol{E}-\boldsymbol{F}$. Details of the first and second lateral branches. $\boldsymbol{G}$. Apical cells. $\boldsymbol{H}$. Rhizoidal filaments formed by some basal cells of the main axis. I-J. Branchlet with sessile and ovoid tetrasporocystes developing adaxially. Scale bar represents $75 \mu \mathrm{m}$. 
(disposition adaxiale) des ramules supérieures en position axiale, 1-2 par cellules, ils mesurent de 54 à $63 \mu \mathrm{m}$ de haut et de 37 à $43 \mu \mathrm{m}$ de large (fig. 2 I-J).

Discussion. Par l'ensemble de ses caractères, tels que la disposition et les dimensions des tétrasporocystes, le type de ramification et le diamètre de l'axe principale, notre échantillon se différencient nettement de Aglaothamnion byssoides (Arnott ex Harvey) C. F. Boudouresque \& M. M. PerretBoudouresque, chez qui le diamètre de l'axe principale est toujours entre $75 \mu \mathrm{m}$ et $150 \mu \mathrm{m}$, le diamètre des cellules apicales est entre 10 $\mu \mathrm{m}$ et $14 \mu \mathrm{m}$. La disposition et les dimensions des tétrasporocystes, ainsi que le type de ramification semblent bien conformes à la description de Maggs \& Hommersand (1993) et aux descriptions et figures publiées par Secilla (2012).

Aglaothamnion pseudobyssoides a été considérée comme un synonyme de $A$. byssoides par Miranda (1932). Mais Halos (1965) et L'Hardy-Halos \& Rueness (1990) ont montré que $A$. pseudobyssoides se différencient de $A$. byssoides par plusieurs caractéristiques morphologiques comme il est mentionné ci-dessus.

Écologie et phénologie. Cette espèce a été récoltée comme épiphyte de Codium tomentosum var. mucronatum, qui se développe sur les rochers à basse mer, parfois fixée au bord des cuvettes de la zone littorale moyenne et inférieure.

Aglaothamnion pseudobyssoides se développe sur des cailloux et épiphyte sur diverses algues notamment sur Codium tomentosum Stackhouse, C. vermilara (Olivi) Delle Chiaje, Gelidium corneum (Hudson) J. V. Lamouroux, Gracilaria multipartita (Clemente) Harvey, Pterosiphonia complanata (Clemente) Falkenberg, Corallina officinalis Linnaeus, Pleonosporium borreri (Smith)
Nägeli, Pterosiphonia ardreana Maggs \& Hommersand, Cladophora lehmanniana (Lindenberg) Kützing; épizoïques sur Balanus et dans les sites protégés (Ardré, 1971; Maggs \& Hommersand, 1993; Secilla, 2012). Les tétrasporocystes ne sont produits qu'entre juillet et le début de septembre (Maggs \& Hommersand, 1993). Selon Secilla (2012) cette espèce a été rencontrée stérile en avril.

\section{RÉFÉRENCES BIBLIOGRAPHIQUES}

ARAÚJO, R., I. BÁRBARA, M. TIBALDO, E. BERECIBAR, P. DÍAZ-TAPIA, R. PEREIRA, R. SANTOS \& O. I. SOUSA-PINTO -2009Checklist of benthic marine algae and cyanobacteria of northern Portugal. Bot. Mar. 52: $24-46$

ARDRÉ, F. -1971- Contribution à l'étude des algues marines du Portugal. II. Écologie et chorologie. Centre d'Études et de Recherches Scientifiques. Biarritz 8: 359-574.

BÁRBARA, I. -2009- Algas Bentónicas Marinas y Salobres de Galicia: Iconografias y Claves de Identificación. Facultad de Ciencias, Universidad de A Coruña, 420pp

BÁRBARA, I., J. CREMADES, S. M. CALVO, C. LÓPEZ-RODRÍGUEZ \& J. DOSIL -2005Checklist of the benthic marine and brackish Galician algae (NW Spain). Anales del Jard. Bot. Madrid 62: 69-100.

BENHISSOUNE, S., C. F. BOUDOURESQUE \& M. VERLAQUE -2002a- A Checklist of the Seaweeds of the Mediterranean and Atlantic Coasts of Morocco. II. Phaeophyceae. Bot. Mar. 45: 217-230.

BENHISSOUNE, S., C. F. BOUDOURESQUE \& M. VERLAQUE -2001- A checklist of marine seaweeds of the Mediterranean and Atlantic coasts of Morocco I. Chlorophyceae Wille s. I. Bot. Mar. 44: 171-182.

BENHISSOUNE, S., C. F. BOUDOURESQUE, M. PERRET-BOUDOURESQUE \& M. VERLAQUE -2002b-A Check-List of Seaweeds of the Mediterranean and Atlantic Coasts of Morocco. III. Rhodophyceae (Excluding Ceramiales). Bot. Mar. 45: 391-412.

BENHISSOUNE, S., C. F. BOUDOURESQUE, 
M. PERRET-BOUDOURESQUE \& M. VERLAQUE -2003-A Check-List of Seaweeds of the Mediterranean and Atlantic Coasts of Morocco. IV. Rhodophyceae-Ceramiales. Bot. Mar. 46: 55-68.

BERDAY, N. -1989-Contribution á l'étude écologique du phythobentbos de la zone littorale de la région d'EL Jadida. Doctorat de 3éme cycle. Fac. Sc. Rabat.

BERMEJO, R., J. L. PÉREZ-LLORENS, J. J. VERGARA \& I. HERNÁNDEZ -2012- Notas corológicas del macrofitobentos marino de Andalucía (España). X. Acta Bot. Malacitana 37: 163-218.

BERECIBAR ZUGASTI. E. -2011- Long-term changes in the phytogeography of the Portuguese Continental Coast. Thesis Universiry of Algarve. $265 \mathrm{pp}$.

BOUXIN, H. \& A. H. DIZERBO -1971- Les algues de l'Archipel des Glenan (Finistère). Bot. Rhedonica Ser. A. 10: 199-226.

CAVASSILLAS, B. -1963-Étude morphologique, écologique et floristique $d u$ bassin $D>E l$ Harhoura. Mémoire de la Société des Sciences naturelles et physiques du Maroc. Bot., nouv. Ser., ${ }^{\circ} 3$. Rabat. 154 pp.

CIRES RODRÍGUEZ, E. \& C. CUESTA MOLINER -2010- Checklist of benthic algae from the Asturias coast (North of Spain). Boletín Ciencias Naturales I. D. E. A., 51: 135-212.

DANGEARD, P. -1949- Les algues marines de la côte occidentale du Maroc. Le Botaniste 34 : 89-189.

DANGEARD, P. -1957- Observations écologiques sur les algues du Maroc atlantique. Paris : Colloques internationaux du C.N.R.S. 276 pp

SMIOLE, J.P. -1962- Recherche sur la végétation marine d'une zone littorale au sud de Rabat. Tray. I. Sc. Sér. Bot. 25.

FELDMANN, J. -1955- La zonation des algues sur la côte atlantique du Maroc. Bull. Soc. Nat. et Physique 35, $1^{\text {ère }}$ trimestre. Maroc. 9-18.

GAYRAL, P. -1958- Algues de la côte atlantique marocaine: Société des Sciences naturelles et physiques du Maroc. Rabat. 523 pp.

GAYRAL, P. -1961- Liste commentée des algues marines nouvelles pour le Maroc reconnues depuis 1949. Rabat: Société des Sciences naturelles et physiques du Maroc, 41: 1-18.

GAYRAL, P. -1966- Les algues des côtes françaises
(Manche et Atlantique). Notions fondamentales sur la biologie, l'écologie et la systématique des algues marines: Doin, Deren et Cie Besançon. Paris. 632 pp.

GOROSTIAGA, J. M., A. SANTOLARIA, A. SECILLA, C. CASARES \& I. DÍEZ -2004Check-list of the Basque coast benthic algae (North of Spain). Anales Jard. Bot. Madrid 61: 155-180.

GRANJA, A., J. CREMADES \& I. BARBARA -1992- Catálogo de las algas bentónicas marinas de la Ría de Ferrol (Galicia, N.O. de la Peninsula Ibérica) y consideraciones biogeograficas sobre su flora. Nova Acta Cient. Compostel. (Biología) 3: 3-21.

GUIRY, M. D. \& G. M. GUIRY -2014- AlgaeBase. World-wide electronic publication, National University of Ireland, Galway. http://www. algaebase.org; searched on 1 July 2014.

HALOS, M. T. -1965- Sur trois Callithamniées des environs de Roscoff. Cah. Biol. Mar. 6: 117-134.

HAMEL, G. -1931- Chlorophycées des côtes Françaises. I. Rouen. XV+168pp.; II: 1-58. Rev. Algol. 1-5 (1925-1931).

HARDY, F. G. \& M. D. GUIRY -2003- $A$ check-list and atlas of the seaweeds of Britain and Ireland. British Phycological Society. London. 435 pp

L'HARDY-HALOS, M. T. \& J. RUENESS -1990Comparative morphology and crossability of related species of Aglaothamnion (Rhodophyta). Phycologia 29: 351-366.

MAGGS, C. A. \& M. H. HOMMERSAND -1993-Seaweeds of the British Isles. Volume 1. Rhodophyta. Part 3A. Ceramiales. British Museum (Natural History). London. 107-110.

MARTÍNEZ-GIL, M., GALLARDO, T., P. DÍAZ \& I. BÁRBARA-2007-Aportación al conocimiento de las algas marinas bentónicas del litoral comprendido entre el estuario del Río Quejo y Punta de la Mesa, Noja, Cantabria, España. Bot. Complut. 31: 41-53.

MIRANDA, F. -1932- Algues marines des côtes de la Manche.Rev. Algo. 6: 281-292.

PEÑA, V. \& I. BÁRBARA -2002- Caracterización florística y zonación de las algas bentónicas marinas del puerto de A Coruña (NO Península Ibérica).Nova Acta Cient. Compostel. (Biología) 12: 35-66.

PEÑA, V. \& I. BÁRBARA-2008- Maërl community in the northwestern Iberian Peninsula: a review 
of floristic studies and long-term changes. Aquat. Conserv. 8: 339-366.

RIADI, H. \& M. KAZZAZ -1998- Inventaire bibliographique des algues benthiques du littoral Marocain I. Chlorophyceae et Phaeophyceae. Acta Bot. Malacitana 23: 23-41.

RODRÍGUEZ-PRIETO, C., BALLESTEROS, E., F. BOISSET \& J. AFONSO CARRILLO -2013- Guía de las Macroalgas y Fanerógamas Marinas del Mediterráneo Occidental. Omega, A. A. Barcelona. $656 \mathrm{pp}$.

SALAHDDINE, N. V. -1982- Étude quantitative et qualitative du macrophytobenthos d'un secteur (Rabat-Bouznika) du littoral océanique marocain. Mémoire de 2éme cycle, I. N. A. V. Hassan II, Rabat.
SECILLA, A. -2012-La familia Ceramiaceae sensu lato en la costa de Bizkaia. Bilbao: Servicio Editorial de la Universidad del País Vasco (18): 369 pp.

TAHI, M. -1983-Étude qualitative et quantitative du macrophytobenthos diun secteur (SaléBouknadel) du littoral atlantique marocain. Mémoire de fin d'étude, option halieutique I. N. A. V. Hassan II. Rabat.

VALENZUELA MIRANDA, S. -2001- Catálogo de las algas recogidas por F. Miranda en la Ría de Pontevedra (NO de España). Nova Acta Cient. Compostel. (Biología) 11: 5-39.

VALENZUELA MIRANDA, S. -2005-Catálogo de las algas recogidas por Faustino Miranda en las costas atlánticas de Francia. Nova Acta Cient. Compostel. (Biología) 14: 5-12. 